\title{
RICHARD MEIER: ORGANIZACIÓN RACIONAL, ESTRUCTURALISMO ESPACIAL Y LUZ
}

\section{RICHARD MEIER: RATIONAL ORGANIZATION, SPATIAL STRUCTURALISM AND LIGHTING}

FECHA DE RECIBO: OCTUBRE 31, 2012

FECHA DE ACEPTACIÓN: DICIEMBRE 20, 2012

MAURICIO CABAS GARCÍA

Especialista en diseño arquitectónico de la Universidad Autónoma del Caribe. Docente de la Corporación Universidad de la Costa-CUC. mcabas1@cuc.edu.co.

\section{RESUMEN:}

El siguiente artículo pretende generar una reflexión acerca del concepto arquitectónico que maneja el arquitecto Richard Meier y tiene como objetivo analizar su proceso de creación y diseño del espacio y el manejo del volumen. Este artículo analiza el manejo que le da al espacio arquitectónico este arquitecto y la creación de una espacialidad única basada en figuras geométricas, retículas, proporciones precisamente calculadas con alto grado de racionalidad integrando perfectamente la luz y el color blanco y dándole un alto grado de expresividad y espiritualidad; de igual manera se pretende explicar cómo su estilo blanco se ha venido desarrollando desde muchos años atrás basado en los arquitectos como Walter Gropius, Alvar Aalto, Mies van der Rohe pero sobre todo en el vocabulario y purismo geométrico de Le Corbusier.

\section{PALABRAS CLAVE:}

Concepto de diseño, Proporción, Luz, Espiritualidad, Estilo, Racionalidad, Volumen, Blanco.

\section{ABSTRACT:}

The following article is intended to generate a reflection on the architectural concept handled by the architect Richard Meier, and aims to analyze about his creative process and design of space and volume management. This article discusses the management that gives the architect to the architectural space and the creation of a unique spatiality based in geometric figures, grids, precisely calculated proportions, high degree of rationality seamlessly integrating light and white, and with a high degree of expressiveness and spirituality, Just as it is intended to explain how white style has been developing for many years based on the architects as Le Corbusier, Walter Gropius, Alvar Aalto and Mies van der Rohe.

\section{KEYWORDS:}

Concept Design, Proportion, Light, Spirituality, Style, Rationality, Volume, White. 


\section{INTRODUCCIÓN}

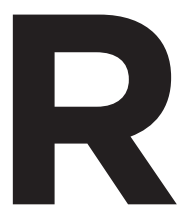

ichard Meier es uno de los más importantes y famosos arquitectos de las últimas décadas, ha llegado a diseñar obras de gran maestría, belleza y racionalidad en las que mezcla formas geométricas con espacios complejos bien articulados. (Barbosky, 2001). La utilización recurrente del blanco, de volúmenes puros, de un manejo radical de la geometría, la racionalización del diseño y el manejo e interpretación del lugar, le ha permitido mantener un estilo propio y que sus obras sean bien reconocidas. Este estilo bastante sobrio ha generado grandes elogios pero también grandes críticas.

Richard Meier es un arquitecto que valora la historia, es estudioso de la misma, observa cada obra de arquitectura y aprende de ella y de su experiencia en el espacio, como lo que el mismo explica sobre el museo Guggenheim de Nueva York diseñado por Frank Lloyd Wright. Del cual reconoció que el espacio interior del museo se aprecia de manera diferente dependiendo de donde se está ubicado, la rampa permite un recorrido que genera distintas perspectivas y la entrada de luz llena el espacio del atrio central dándole una interpretación del mismo. (Meier, Entrevista con Richard Meier, 1994)

Meier, nacido en 1934, empezó su carrera diseñando viviendas unifamiliares en los Estados Unidos de América y las cuales tenían un alto grado abstracción formal, como la Casa Smith, la casa Douglas y la casa Helmick entre otras. Tiempo después y luego de recibir encargos en Europa y de ganar numerosos e importantes concursos pasó a convertirse en un arquitecto conocido por sus obras de carácter público. En el presente artículo se pretenderá analizar cuatro de las obras más reconocidas diseñadas por este arquitecto con el fin de explicar más a fondo los conceptos del mismo.

\section{RELACIÓN CON LO NATURAL}

El espacio arquitectónico diseñado por Richard Meier no pretende contar con un ideal orgánico; más bien intenta involucrarse con su contexto de manera reflexiva. Generalmente el uso que hace de las formas geométricas blancas se podría considerar una victoria sobre la naturaleza, aunque para Meier los principios con los que organiza sus diseños son completamente complementarios con ella. Meier (1994) es consciente y exclama que hacer arquitectura es un acto que no es natural, es una declaración acerca de lo que verdaderamente hacen los arquitectos, intervenir lo natural y critica a Frank Lloyd Wright por el concepto de la naturaleza de los materiales y como éste pensaba que se debían utilizar materiales naturales, pero al momento de tomar madera para la construcción, ya esa madera no está viva, se convierte en algo inerte como cualquier otro material de construcción. De igual manera la arquitectura debería colaborar con las per- cepciones y sensaciones que genera la naturaleza cambiante. Es por esto el claro contraste de los proyectos de Meier y el background o telón de fondo del contexto natural en los que se encuentran. Como si el verde de la grama y el azul del cielo hicieran resaltar el blanco de los volúmenes geométricos y viceversa. La espacialidad y el manejo de la luz son el gran aporte de Meier a la arquitectura, un espacio inmaculado, lleno de iluminación y complementado por transparencias. Ha generado una manera propia de interpretar los conceptos básicos de los grandes genios de la arquitectura, Mies Van der Rohe y de Le Corbusier y crear un estilo propio muy reconocido.

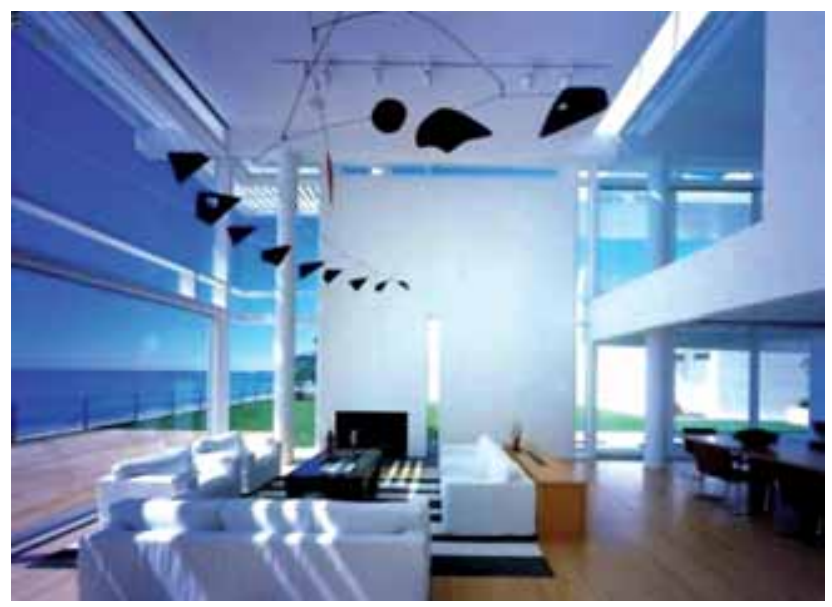

Casa de Playa al Sur de California. Richard Meier \& Partners. Fotografia: Scott Frances/ESTO

\section{MANEJO DE LA LUZ Y EL BLANCO}

Las obras de Meier están llenas de estímulos para los sentidos, aunque se piense lo contrario por su apariencia blanca y geométrica. Esto debido al manejo casi espiritual de la luz en esas edificaciones. La luz entra a las obras de Richard Meier como un caudal de agua desbordante, lo que le permite sentir sensaciones distintas a lo largo del día. Jodidio (2010) expresa que la arquitectura de este arquitecto cambia constantemente gracias a las nubes, la posición del sol en el cielo, el cambio de estaciones y a la luna.

En las construcciones residenciales hechas por Meier, en las cuales no hay ningún uso del color, el sol y el cielo son los encargados de incorporar los toques de color tanto en el exterior como en el interior. Por el contrario, en las noches, el sublime manejo de la luz artificial hace que esas viviendas resplandezcan desde su interior, y se conviertan en faros en la oscuridad. Este manejo de la luz natural y de la iluminación artificial le da un toque de calidez a esta arquitectura que diera la impresión de ser algo fría. 
ARTE \& DISEÑO, ISSN: 1692-8555, Vol. 10 Núm. 2, Julio - Diciembre de 2012 Richard Meier: organización racional, estructuralismo espacial y luz, págs. 5-11

\begin{abstract}
"Si es cierto que Ludwig Mies Van der Rohe afirmó una vez que, <<Dios está en los detalles >>, de la arquitectura de Richard Meier sería posible decir que Dios está en los números. Más que cualquier otro arquitecto contemporáneo, Richard Meier ha impuesto un estilo, casi invariablemente determinado por las retículas y por las proporciones calculadas con precisión, aunque esos elementos aritméticos no son los únicos componentes predecibles de sus diseños." (Jodidio, 2010)
\end{abstract}

Richard Meier adoptó el blanco a mediados de la década de 1960, con el fin de enfatizar el espacio y la luz. De igual manera, el blanco hacía pensar que el estilo y el purismo de Le Corbusier no era algo involuntario, al igual que el claro vocabulario geométrico que maneja en sus proyectos. La arquitectura de Meier se centra en dos temas recurrentes: el blanco como textura y la abstracción como lenguaje. Sus obras evidencian madurez, elegancia, equilibrio y pureza como resultado de la abstracción, de la experiencia y de la búsqueda de la belleza. Los proyectos de Richard Meier son una manifestación de la poesía en la arquitectura. El mismo arquitecto conceptúa al respecto, diciendo:

"El blanco es el emblema efimero de un movimiento perpetuo. El blanco está siempre presente, pero nunca es el mismo: brillante e inestable durante el día; plateado y efervescente bajo la luna llena del año nuevo. Entre el océano de la consciencia y la inmensa materialidad de la tierra, se extiende esa línea siempre cambiante del blanco. El blanco es luz, el medio de la comprensión y del poder transformador." (Meier, 2012)

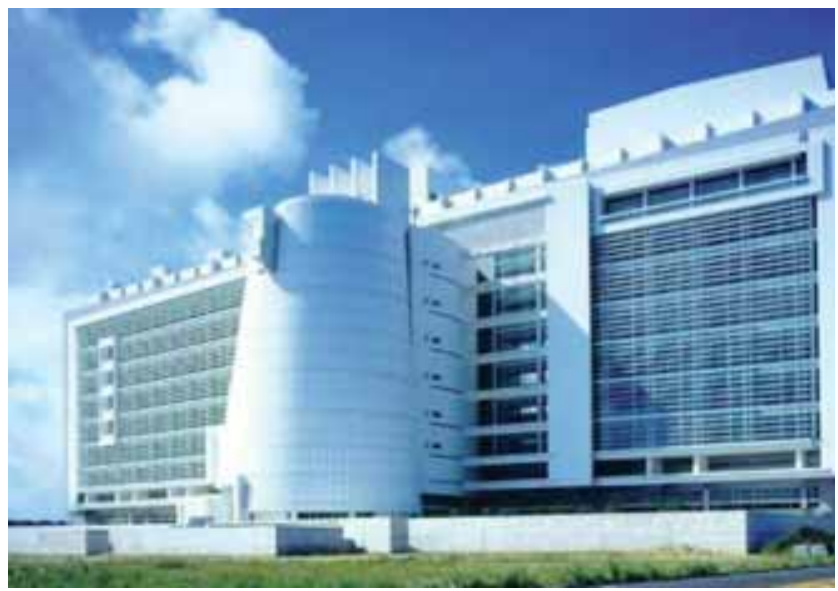

Corte de Justicia de Islip. Richard Meier \& Partners. Fotografia: Scott Frances/ESTO

Para Richard Meier, la escogencia del blanco no era solo una cuestión de moda o de simplificar su arquitectura, todo lo contrario; para él, el blanco es significado de pureza y claridad así como la representación de algo perfecto. El blanco hace resaltar todos los detalles de una edificación, hay que saberlo trabajar, y además permite jugar con la volumetría por medio de las sombras.

Algo que deja muy claro este arquitecto es que la arquitectura debe inspirar, debe elevar el espíritu, debe ser un elemento que nutra la mente y el cuerpo, y se convierte en el escenario de todas las artes. (Meier, 2011).

\section{RACIONALIDAD DEL DISEÑO, ESTRUCTURALISMO ESPACIAL E HIBRIDACIÓN VOLUMÉTRICA}

Richard Meier tiene como fin al momento de diseñar, crear espacios coherentes, comprensible y funcionales. Así mismo, busca generar un espacio donde la luz configure el entorno creando un sentido de bienestar, que logra un alcance espiritual. Su proceso de diseño se basa en sistemas de relaciones muy racionales, Gössel y Leuthäuser (1991) afirman que Richard Meier utiliza tres pares de relaciones que son: programa y situación, entrada y circulación, estructura y caparazón. De igual manera el entorno configura los ejes y las perspectivas que ordenan la volumetría del edificio.

"Creo que la responsabilidad del arquitecto dice Richard Meier- es realmente la de crear un sentido del orden, del lugar, de las relaciones. Esas ideas son inherentes a la arquitectura $y$, por tanto, la precisión o las relaciones son muy importantes para mí al aclarar en lo posible las ideas y relaciones y al crear un sentido del orden. No estoy interesado en crear caos; eso lo dejo para otros". (Jodidio, 2010)

La racionalidad en el diseño de Meier está enmarcada en el proceso de pensar y analizar todas las alternativas posibles teniendo en cuenta los sistemas de relaciones anteriormente mencionadas. La organización espacial fundamentada en los espacios de circulación mantiene un orden y una claridad constante. Tal vez uno de los puntos altos en el proceso de diseño de Meier es el uso y la forma de materializar el sentido común. El mismo arquitecto compara la organización de los espacios y el programa de sus obras con el proceso creativo del collage, ya que esto es un ejercicio de agilidad mental, es un ejercicio de raciocinio y de composición. (Meier \& Logan, Museu d'art contemporani de Barcelona, 2010)

Meier desarrolla un repertorio de volúmenes curvos y rectilíneos en casi todos sus proyectos, pero dejando que el proyecto mismo se repliegue por medio de rampas que generalmente es una invitación extendida y abierta a los peatones.

Generalmente las obras de Richard Meier adquieren una apariencia de perfección, alejadas de su entorno, al menos desde una perspectiva externa. El cilindro se ha convertido en parte importante en las obras de Meier, y la utilización del mismo, ya sea en su 
ARTE \& DISEÑO, ISSN: 1692-8555, Vol. 10 Núm. 2, Julio - Diciembre de 2012 MAURICIO CABAS GARCÍA

forma pura o con variaciones tipo cono, adquirió mucha relevancia en sus diseños así como la combinación de composiciones asimétricas de muros curvos, lineales y mixtos. Las variaciones volumétricas que Meier ha experimentado a lo largo de su carrera le han permitido equilibrar las masas y pesos de sus edificios, llegando a crear volúmenes híbridos elaborados y no tan elaborados mezclando cubos y cilindros que contrastan con mucha gracia con su entorno, y que juegan y encajan con largos muros pantalla como sucede en el Museo de Arte Moderno de Barcelona. (Rykwert, 1999)

\section{LAS OBRAS}

\section{- Casa Smith}

La casa Smith está situada en un lote de $6070 \mathrm{~m}^{2}$ con vistas hacia el estrecho de Long Island y en ella se logran apreciar los conceptos claros que maneja Richard Meier. Esta casa está revestida en madera pintada de blanco, y posee un vocabulario moderno y un carácter minimalista, pero Meier logra darle una sensación de confort, de luminosidad y de amplitud apartado completamente de los paradigmas funcionalistas de los inicios del movimiento moderno. (Jodidio, 2010)

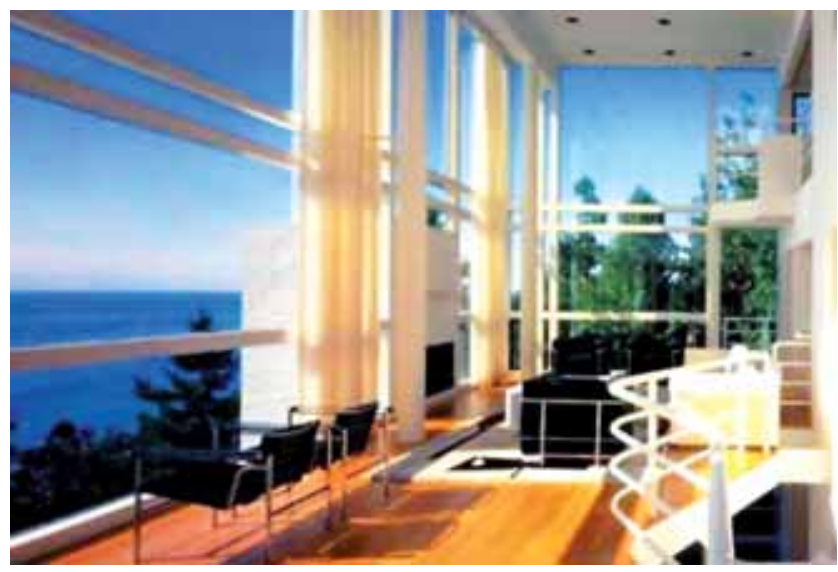

Casa Smith. Richard Meier \& Partners. Fotografia: Scott Frances/ESTO

La Casa Smith, cuenta con una espacialidad única y deslumbrante. Contiene un puente de acceso, una fachada cerrada por el lado de la entrada y una abierta frente al agua, y una sala de techo alto y un generoso ventanal. La organización espacial de la casa se basa en la separación de los espacios privados y los espacios públicos. El área privada está localizada cerca de la entrada frente al terreno, los arboles y la vía cercana. Una serie de espacios de servicio como guardarropas y depósitos son los articuladores entre las zonas y al mismo tiempo las separan. Los espacios privados están distribuidos en tres niveles detrás de una fachada casi cerrada que cuenta con unas cuantas ventanas colocadas para beneficiar la entrada de luz y las visuales. Los espacios públicos o sociales están en la parte opuesta del acceso, en la parte de la casa que tiene vista al mar. Esta zona social está distribuida en tres niveles y está enmarcada por grandes ventanales. El concepto de transparencia se ve intensificado por el paisaje y la vista al mar.

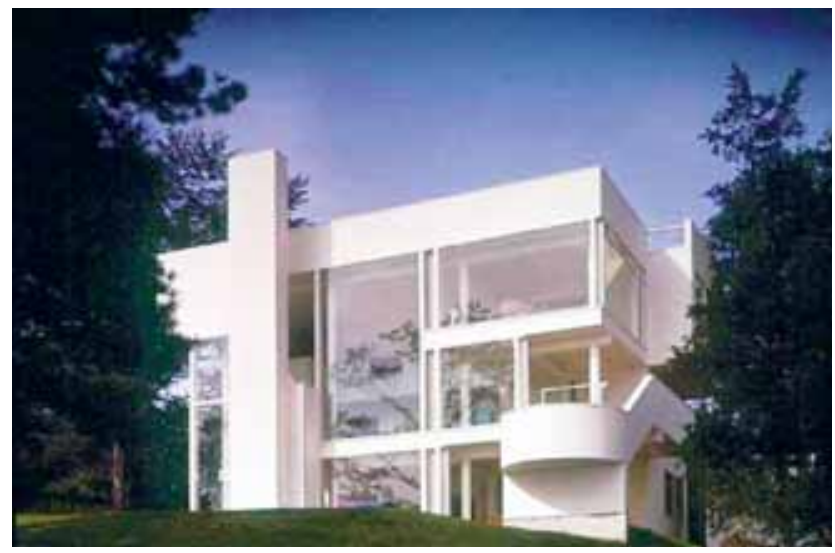

Casa Smith. Richard Meier \& Partners. Fotografia: Scott Frances/EST0

\section{- El Ateneo}

La arquitectura de Richard Meier evolucionó de gran forma con el diseño del Ateneo de New Harmony, ya que con esta obra se empezaron a identificar mucho más claramente el lenguaje y los conceptos arquitectónicos que caracterizarían su estilo al momento de diseñar edificios de carácter público. El proyecto se encuentra aislado y elevado del nivel del suelo para prevenir cualquier inundación debido a la cercanía del río Wabash. El Ateneo cuenta con lenguaje moderno pero con un estilo náutico y una prominente rampa de acceso como si fuese un muelle. Desde el punto de vista del concepto del espacio arquitectónico interior se plantea un movimiento continuo con la rampa como elemento importante y definitivo. Los espacios se definen por dos retículas giradas 5 grados entre sí. La estética del edificio está concebida por el revestimiento de paneles de porcelana esmaltada dividida en cuadrículas. (Jodidio, 2010)

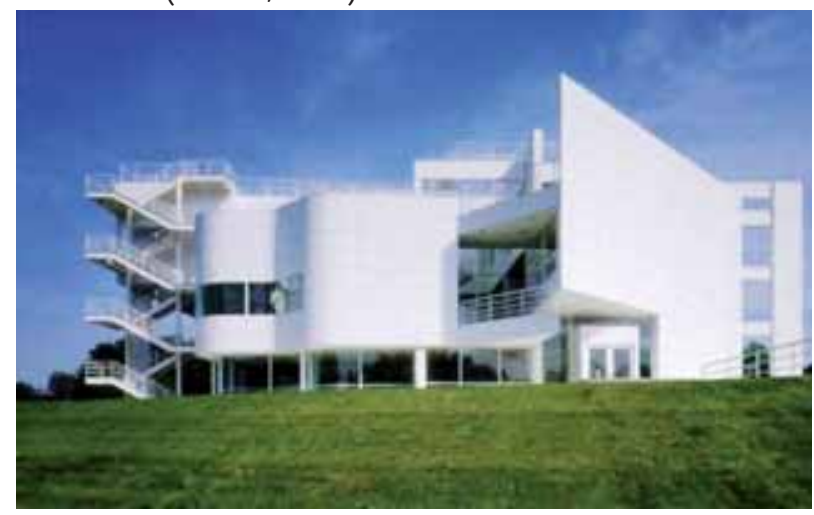

El Ateneo. Richard Meier \& Partners. Fotografia: Ezra Stolller/ESTO

Sobre la retícula ortogonal que actúa como base del volumen del Ateneo, se acomodan las necesidades del programa. En primer piso son tres zonas, de las cuales predomina el auditorio cerrado ubicado del lado contrario a las mejores visuales. Las otras dos zonas se ajustan a las visuales y al eje de circulación del acceso. 
ARTE \& DISEÑO, ISSN: 1692-8555, Vol. 10 Núm. 2, Julio - Diciembre de 2012 Richard Meier: organización racional, estructuralismo espacial y luz, págs. 5-11

Una rampa colocada al lado del auditorio enfatiza la horizontalidad de una de las zonas. Esta misma rampa, en su segundo tramo, se acomoda a un eje girado que define, al prolongarse, la aproximación al edificio desde el río y desde el pueblo de New Harmony. El eje girado se transforma en un plano o pantalla que organiza el espacio interior y la escalera. Esta rotación no es ningún capricho está justificada por la orientación del trazado del pueblo con la orilla del río. Arriba del primer cuadrado se sitúa otro de tamaño superior pero girado considerablemente, lo que aumento los efectos de oblicuidad. En ese segundo cuadrado se crea una pantalla que queda perpendicular al camino peatonal dándole mayor jerarquía al acceso. (Baker, 1998) Con el Ateneo de New Harmony, Meier logra una espacialidad que se relaciona perfectamente con el exterior, con giros en su planta que permiten generar distintas perspectivas dando sensación de dinamismo y permitiendo la entrada de luz natural.

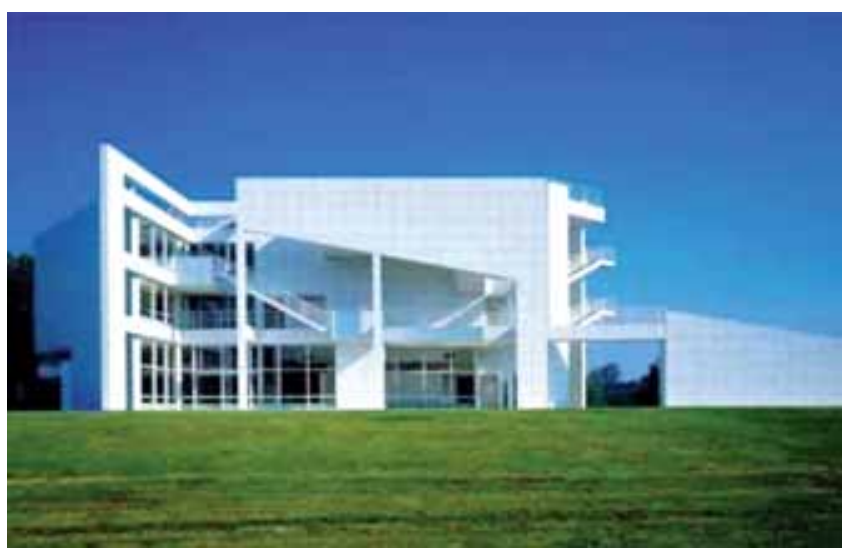

El Ateneo. Richard Meier \& Partners. Fotografia: Ezra Stolller/ESTO

\section{- El Centro Getty}

Sin duda, puede considerarse que el mayor proyecto diseñado por Richard Meier es el Getty Center, ubicado en la ciudad de Los Ángeles, y el que contribuyó en gran medida a definir su reputación. Este proyecto cuenta con $88.000 \mathrm{~m} 2$ y está situado en un lote de 44,5 hectáreas en la cima de una colina, que domina todo su entorno. Este proyecto tiene reminiscencias clásicas, no desde lo estético sino desde el manejo del espacio público y la organización de los edificios. El mismo Richard Meier (2010) comentó que el Getty Center parece o pude ser comparada con una estructura aristotélica en la forma como se relaciona con el paisaje y el lugar. El proyecto dialoga con el entorno, se funden como un todo. De igual manera el proyecto se asemeja a la Villa del emperador Adriano debido al orden y secuencia de los espacios, la utilización de muros gruesos y la compenetración con el paisaje. Estas características parecidas a la Villa de Adriano en Tivoli, que demuestra la afinidad que siente Meier por el pasado, ya sea del siglo pasado o incluso anterior.

El Getty Center, a diferencia de todos los anteriores proyectos de Meier no es blanco debido a la normativa de la ciudad de Los Ángeles que determinaba específicamente que los edificios no podían ser blancos, así que Meier se vio obligado a buscar un revestimiento. Escogió un mármol travertino italiano de textura rústica, lo que significaba un drástico y radical cambio de cualquiera de los suaves revestimientos que había elegido en el pasado.

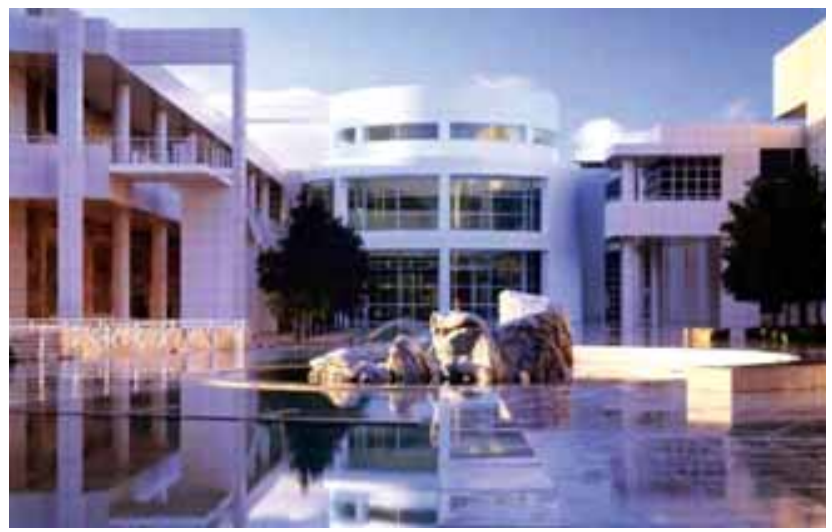

Getty Center. Richard Meier \& Partners. Fotografia: Scott Frances/ESTOMicahel Rutchik/Mudhaus

Este mármol travertino tiene una superficie áspera y de apariencia pesada, lo que contrasta con el concepto utilizado por Meier en proyectos anteriores, en los cuales la perfección y las superficies casi sin texturas y livianas son evidentes. La idea de una villa de la antigua Grecia o Roma, se intensifica debido a los muros de contención en las laderas escarpadas y se acerca al concepto del edificio como lugar relacionado con las culturas clásicas. Esto no es por coincidencia o capricho, la colección del museo Getty está conformada en su mayoría de esculturas griegas y romanas. (Jodidio, 2010)

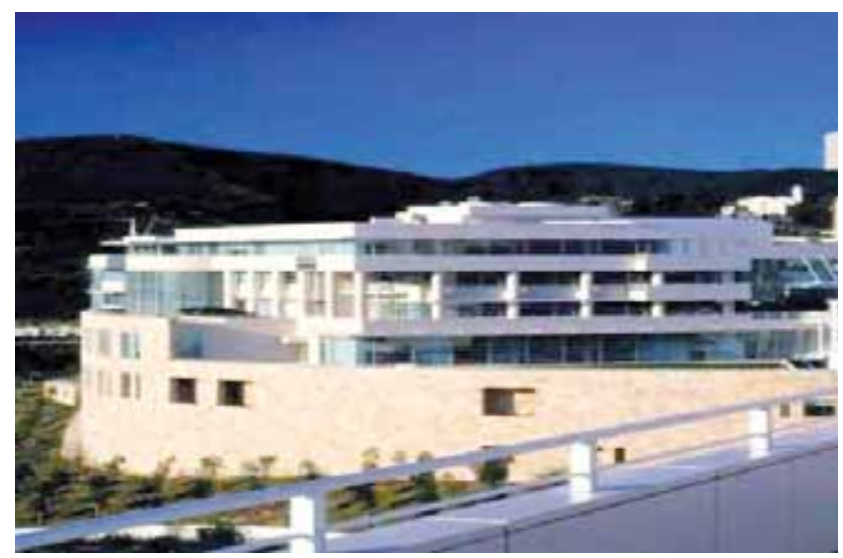

Getty Center. Richard Meier \& Partners. Fotografia: Scott Frances/ESTO, Micahel Rutchik/Mudhaus

Meier implanta el proyecto de una manera que juega con la topografía escarpada del lugar, respondiendo, de igual manera, al programa impuesto por el cliente, que deseaba tener zonas independientes, como el museo, el centro de historia de arte y 
ARTE \& DISEÑO, ISSN: 1692-8555, Vol. 10 Núm. 2, Julio - Diciembre de 2012 MAURICIO CABAS GARCÍA

humanidades, el instituto de conservación y el centro de estudios artísticos. Los seis edificios independientes que conforman el complejo tenían que ser multipropósito, pero como complejo en si tiene que ser una unidad. Cada edificio tiene su propia identidad dentro de un solo conjunto. (Jodidio, 2010) Los principios fundamentales de diseño utilizados en este proyecto fueron la relación con el contexto, la geometría evidenciada en dos retículas giradas 22.5 grados entre sí como base para la organización espacial, la estructura, las circulaciones, la configuración de los espacios exteriores y el paisajismo. El complejo sigue conceptos de flexibilidad, invención, calidez y amplitud conjugados con ideales europeos como la permanencia, historia y especificidad. Los materiales utilizados reafirman la imagen de solidez. Como una roca en el paisaje. Este tipo de arquitectura integra la escala humana con la grandeza de los espacios cívicos, la simplicidad decorativa con la riqueza de los acabados y la innovación técnica con un alto respeto de los preceptos históricos. (Meier, Richard Meier Architect 3, 1999)

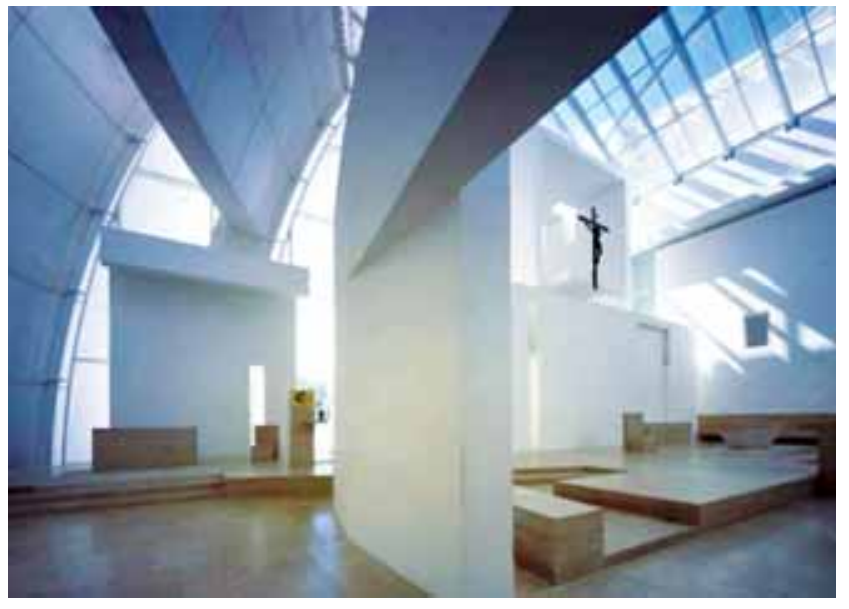

Iglesia del Jubileo. Richard Meier \& Partners. Fotografia: Scott Frances/ARCAID

La iglesia católica del Jubileo (1996-2003) fue la primera construcción que realizó Roma, lo que resultaba extraño era que se le encargara este tipo de proyecto a un arquitecto de otra religión, debido a la considerable importancia simbólica del la obra. Meier proyecto formas similares a velas, pero geométricamente concebidas, la estructura se extiende sobre un cuadro desplazado y cuatro círculos. Se generan tres armazones en forma de concha que determinan las líneas primarias del templo y están diseñadas teniendo en cuenta otras tres circunferencias de igual radio en una discreta referencia a la Santísima Trinidad. Richard Meier logra demostrar con el uso del blanco en la totalidad del espacio interior de la Iglesia del Jubileo que puede crear un espacio humano bañado de luz que inspira y genera reflexión, es un espacio que da la sensación de divinidad con un lenguaje sencillo. (Jodidio, 2010)

\section{CONCLUSIONES}

La maestría formal, racional y estética que ha logrado Richard Meier, es producto de la consistencia da la aplicación, a lo largo de su carrera, con una gran convicción de los conceptos arquitectónicos en exploración del contraste entre la ortogonalidad, los giros axiales y la sinuosidad. Su arquitectura es una síntesis de geometrías depuradas, limpias, blancas muy influenciado por Le Corbusier, utilizando un vocabulario arquitectónico como lo son una estructura independiente, plantas libres y grandes ventanales que permiten gran luminosidad interior y al mismo tiempo una relación estrecha con el exterior. Ujueta (2002) comenta que la arquitectura de Richard Meier es una arquitectura de gran exquisitez y que solo las personas más adineradas pueden obtenerla, debido al alto costo de sus elementos y a los exclusivos lugares en donde se encuentran.

La arquitectura de Richard Meier es relativamente fácil de leer: consiste en una sintaxis de la arquitectura moderna evolucionada y adaptada a los tiempos contemporáneos. Sus conceptos fundamentales claramente se derivan de arquitecturas anteriores, pero Meier los ha sabido interpretar y reinventar. El manejo de una racionalidad planimétrica regular que no implica simetría, la composición de volúmenes geométricamente puros que no expresan monotonía, el extremado cuidado por los detalles y la utilización del blanco como elemento generador de sensaciones de luminosidad y limpieza. Así mismo, un análisis cuidadoso del entorno le permite crear relaciones insospechadas con la naturaleza, Meier sabe utilizar la naturaleza como lienzo, sabe manipular los reflejos de la luz en sus volúmenes, sabe conjugar el movimiento con lo estático.

En sus cinco décadas de trabajo, Richard Meier se mantiene constante y fresco, fiel a sus principios que definen sus primeras obras. Existe un vínculo directo entre la casa Smith de 1965 y sus últimas obras que se construyen en la actualidad. El lenguaje se ha vuelto más rico, los detalles y las terminaciones más precisas, pero en ambos casos se emplea la misma estética minimalista de planos blancos, ventanales amplios y volúmenes primarios cortados y mezclados llenos de luz natural.

Meier es un modernista empedernido que no se ha dejado distraer de estilos como el postmodernismo o el deconstructivismo, su inspiración viene de lo clásico. (Leonardi, 2011)

Obviamente, Meier aunque tiene raíces modernistas bastante marcadas, logra interpretar tendencias y conceptos de arquitectos completamente distintos a los arquitectos modernistas, lo que demuestra su versatilidad y su consistencia. 
ARTE \& DISEÑO, ISSN: 1692-8555, Vol. 10 Núm. 2, Julio - Diciembre de 2012 Richard Meier: organización racional, estructuralismo espacial y luz, págs. 5-11

\section{REFERENCIAS BIBLIOGRÁFICAS}

Adams, J. (2007). Richard meier reflects on over 40 years of architecture. Contemporary Stone \& Tile Design, 13(3), 24$24,26,29,31$. Retrieved from http://search.proquest.com/docvi ew/210363633? accountid $=43787$

Barbosky, M. (2001). Arquitectura siglo XX. Madrid: Electa.

Gössel, P., \& Leuthäuser, G. (1991). Arquitectura del siglo XX. Colonia: Taschen.

Jodidio, P. (2010). Richard Meier. Colonia: Taschen.

Leonardi, N. (2011). Interview with Richard Meier. The Plan. Architecrture and Technologies in detail , 2-20.

Meier, R. (24 de Mayo de 2011). 3dsign. Recuperado el 25 de Enero de 2013, de http://www.3dsign.es/blog/2011/05/24/ arquitecto-del-dia-richard-meier/

Meier, R. (31 de Mayo de 2012). Cosas de Arquitectos. Recuperado el 25 de Enero de 2013, de El blanco es un emblema efimero de un movimiento perpetuo: http://www.cosasdearquitectos. com/2012/05/el-blanco-es-el-emblema-efimero-de-un-movimiento-perpetuo-meier/

Meier, R. (16 de Mayo de 1994). Entrevista con Richard Meier. (P. Jodidio, Entrevistador)

Meier, R. (1999). Richard Meier Architect 3. Nuava York: Rizzoli International Publications, Inc.

Meier, R. (1992). Richard Meier Arquitecto. Barcelona: Gustavo Gili.

Meier, R., \& Logan, R. (2010). Museu d’art contemporani de Barcelona. Recuperado el 1 de Marzo de 2012, de Las calles mediavales inspiraron la organizacion lineal del MACBA: http://www. macba.cat/PDFs/entrevista_meier_cas.pdf

Rykwert, J. (1999). On Form and Style. En L. Green, Richard Meier Architect 4 (págs. 16-19). Nueva York: Rizzoli International Publications, Inc.

Popham, P. (2004). Richard meier achieves a baroque sense of space and light with concrete construction in the JUBILEE CHURCH in rome, italy ; Architectural Record, 192(2), 100-107. Retrieved from http://search.proquest.com/docview/222117780 ?accountid $=43787$

Ujueta, C. (2002). La Arquitectura en la cultura occidental. . Barranquilla: Ediciones Uniautonoma. 